hope has often been more hype than realization. The story of Marfan syndrome and mutations in the FBN1 gene illustrates that identifying a mutation is only the first step in understanding the molecular pathophysiology of the disorder and that the pathway to treatment may be both complex and full of insights into unexpected areas of biology $(24,25)$. Perhaps in a short time we may see the use of TGF- $\beta$ blockade in preference to $\beta$-(adrenergic) blockade as a more effective treatment of many aspects of Marfan syndrome - and treatment is the ultimate goal of understanding this complex phenotype.

Address correspondence to: Peter H. Byers, Department of Pathology, Box 357470, University of Washington, Seattle, Washington 98195-7470, USA. Phone: (206) 543-4206; Fax: (206) 616-1899; E-mail: pbyers@u.washington.edu.

1. Dietz, H.C., et al. 1991. Marfan syndrome caused by a recurrent de novo missense mutation in the fibrillin gene. Nature. 352:337-339.

2. Sakai, L.Y., Keene, D.R., and Engvall, E. 1986. Fibrillin, a new $350-\mathrm{kD}$ glycoprotein, is a component of extracellular microfibrils. J. Cell Biol. 103:2499-2509.

3. Hollister, D.W., Godfrey, M., Sakai, L.Y., and Pyeritz, R.E. 1990. Immunohistologic abnormalities of the microfibrillar-fiber system in the Marfan syndrome. N. Engl. J. Med. 323:152-159.

4. Lee, B., et al. 1991. Linkage of Marfan syndrome and a phenotypically related disorder to two different fibrillin genes. Nature. 352:330-334.
5. Magenis, R.E., Maslen, C.L., Smith, L., Allen, L., and Sakai, L.Y. 1991. Localization of the fibrillin (FBN) gene to chromosome 15, band q21.1. Genomics. 11:346-351.

6. Milewicz, D.M., Pyeritz, R.E., Crawford, E.S., and Byers, P.H. 1992. Marfan syndrome: defective synthesis, secretion, and extracellular matrix formation of fibrillin by cultured dermal fibroblasts. J. Clin. Invest. 89:79-86.

7. The FBN1 mutations database. http://www.umd. be:2030/.

8. Faivre, L., et al. 2003. In frame fibrillin-1 gene deletion in autosomal dominant Weill-Marchesani syndrome. J. Med. Genet. 40:34-36.

9. Ades, L.C., et al. 2002. Segregation of a novel FBN1 gene mutation, G1796E, with kyphoscoliosis and radiographic evidence of vertebral dysplasia in three generations. Am. J. Med. Genet. 109:261-270.

10. Katzke, S., et al. 2002. TGGE screening of the entire FBN1 coding sequence in 126 individuals with marfan syndrome and related fibrillinopathies. Hum. Mutat. 20:197-208.

11. Sood, S., Eldadah, Z.A., Krause, W.L., McIntosh, I., and Dietz, H.C. 1996. Mutation in fibrillin-1 and the Marfanoid-craniosynostosis (Shprintzen-Goldberg) syndrome. Nat. Genet. 12:209-211.

12. Bona, C.A., et al. 1997. Structure of the mutant fibrillin-1 gene in the tight skin (TSK) mouse. DNA Res. 4:267-271.

13. Eldadah, Z.A., Brenn, T., Furthmayr, H., and Dietz, H.C. 1995. Expression of a mutant human fibrillin allele upon a normal human or murine genetic background recapitulates a Marfan cellular phenotype. J. Clin. Invest. 95:874-880.

14. Judge, D.P., et al. 2004. Evidence for a critical contribution of haploinsufficiency in the complex pathogenesis of Marfan syndrome. J. Clin. Invest. 114:172-181. doi:10.1172/JCI200420641.

15. Putnam, E.A., Zhang, H., Ramirez, F., and Milewicz, D.M. 1995. Fibrillin-2 (FBN2) mutations result in the Marfan-like disorder, congenital contractural arachnodactyly. Nat. Genet. 11:456-458.
16. Corson, G.M., Charbonneau, N.L., Keene, D.R., and Sakai, L.Y. 2004. Differential expression of fibrillin-3 adds to microfibril variety in human and avian, but not rodent, connective tissues. Genomics. 83:461-472.

17. Annes, J.P., Munger, J.S., and Rifkin, D.B. 2003. Making sense of latent TGFbeta activation. J. Cell. Sci. 116:217-224.

18. Nakajima, Y., Miyazono, K., and Nakamura, H. 1999. Immunolocalization of latent transforming growth factor-beta binding protein-1 (LTBP1) during mouse development: possible roles in epithelial and mesenchymal cytodifferentiation. Cell Tissue Res. 295:257-267.

19. Saharinen, J., and Keski-Oja, J. 2000. Specific sequence motif of 8-Cys repeats of TGF-beta binding proteins, LTBPs, creates a hydrophobic interaction surface for binding of small latent TGF-beta. Mol. Biol. Cell. 11:2691-2704.

20. Kaartinen, V., and Warburton, D. 2003. Fibrillin controls TGF-beta activation. Nat. Genet. 33:331-332.

21. Saito, S., et al. 1999. Characterization of mutated protein encoded by partially duplicated fibrillin-1 gene in tight skin (TSK) mice. Mol. Immunol. 36:169-176.

22. Neptune, E.R., et al. 2003. Dysregulation of TGF-beta activation contributes to pathogenesis in Marfan syndrome. Nat. Genet. 33:407-411.

23. Hutchinson, S., et al. 2003. Allelic variation in normal human FBN1 expression in a family with Marfan syndrome: a potential modifier of phenotype? Hum. Mol. Genet. 12:2269-2276.

24. Aoyama, T., Tynan, K., Dietz, H.C., Francke, U., and Furthmayr, H. 1993. Missense mutations impair intracellular processing of fibrillin and microfibril assembly in Marfan syndrome. Hum. Mol. Genet. 2:2135-2140

25. Frischmeyer, P.A., and Dietz, H.C. 1999. Nonsensemediated mRNA decay in health and disease. Hum. Mol. Genet. 8:1893-1900.

26. Kaartinen, V., and Warburton, D. 2003. Fibrillin controls TGF- $\beta$ activation. Nat. Genet. 33:331-332.

\title{
Unbuckling lipodystrophy from insulin resistance and hypertension
}

\author{
Robert A. Hegele ${ }^{1}$ and Todd Leff ${ }^{2}$
}

\begin{abstract}
${ }^{1}$ Robarts Research Institute, London, Ontario, Canada. ${ }^{2}$ Department of Pathology, Wayne State University School of Medicine, Detroit, Michigan, USA.
\end{abstract}
\begin{abstract}
Lipodystrophy and insulin resistance are the core features of human PPAR $\gamma$ deficiency states. Metabolic complications in PPAR $\gamma$ deficiency, such as hypertension, have been considered to be secondary to insulin resistance. However, a new mouse model that expresses the analog of a human PPARG mutation displays minimal lipodystrophy and insulin resistance but rather severe hypertension (see the related article beginning on page 240). Furthermore, the mutant protein appears to directly modulate the renin-angiotensin system in adipose tissue, providing evidence of the pleiotropic effects of PPAR $\gamma$.
\end{abstract}

Nonstandard abbreviations used: angiotensin II (ATII); familial partial lipodystrophy type 3 (FPLD3); metabolic syndrome (MetS); renin-angiotensin system (RAS); type 2 diabetes mellitus (T2DM).

Conflict of interest: The authors have declared that no conflict of interest exists.

Citation for this article:

J. Clin. Invest. 114:163-165 (2004).

doi:10.1172/JCI200422382.
The commonly occurring metabolic syndrome (MetS) is considered to result from complex gene-environment interactions and has been associated with future onset of type 2 diabetes mellitus (T2DM) (1) and both all-cause and cardiovascular mortality (2). MetS is defined clinically according to deviation from threshold values for three or more of five quantitative traits; namely, waist circumference, blood pressure, and plasma concentrations of glucose, highdensity lipoprotein cholesterol, and triglyceride (3). Insulin resistance has long been considered the core biochemical defect linking these metabolic disturbances, which are strongly correlated among and within patients, suggesting the existence of common underlying molecular mechanisms. No molecule is more central to the metabolic and vascular pathways of MetS than PPAR $\gamma$ (4). The effect of altered activity of the PPAR $\gamma$ receptor on whole-body insulin sensitivity has been appreciated for years. For instance, in both mice and humans, activating PPAR $\gamma$ ligands has ben- 


\section{Table 1}

Comparison of selected phenotypes of human lipodystrophies FPLD2 and FPLD3 and the mouse Pparg ${ }^{P 465 L+}$

\begin{tabular}{lc} 
Species & Mutation \\
Human & FPLD2 $($ LMNA) \\
& FPLD3 $($ PPARG $)$ \\
Mouse & Pparg \\
\hline
\end{tabular}

Extent of partial lipodystrophy
Pronounced fat loss on extremities and gluteal region
Moderate fat loss on extremities
Mild fat redistribution

Insulin resistance

Moderate to severe

Severe

Minimal or absent
Hypertension

Moderate to severe

Severe

Severe eficial effects on insulin sensitivity (5). But recent studies of rare patients with lossof-function mutations in PPARG and of mice in which Pparg has been manipulated have shown similarities and discrepancies, which underscore PPAR $\gamma$ 's physiological complexity and its pleiotropic effects.

\section{Mutations in PPAR $\gamma$ cause human lipodystrophy}

In humans, the relationship between PPAR $\gamma$ activity and insulin sensitivity appears to be relatively straightforward: increased PPAR $\gamma$ activity via activating ligands leads to increased insulin sensitivity (5), while reduced receptor activity via germline lossof-function mutations, such as P467L, leads to insulin resistance (6). Human PPARG mutations are associated with the recently identified syndrome familial partial lipodystrophy type 3 (FPLD3, OMIM 604367), which is characterized by relative depletion of subcutaneous fat on extremities along with preservation of central and visceral fat stores. The experiments by Tsai, Maeda, et al. reported in this issue of the JCI (7) show that Pparg ${ }^{P 465 L /+}$ mice - whose genotype is homologous to that of heterozygous PPARG $G^{\text {P467L }}$ patients - also have repartitioning of adipose stores, albeit in a somewhat different pattern compared with that associated with human FPLD3. These findings, together with previous reports (8-11), firmly establish PPAR $\gamma$ deficiency as a cause of lipodystrophy and confirm the key adipogenic role of PPAR $\gamma$.

\section{Disproportionate hypertension in Pparg $^{\text {P465L/+ mice }}$}

The next question is whether lipodystrophy associated with PPAR $\gamma$ deficiency is mechanistically linked with insulin resistance and its complications, particularly hypertension. Among FPLD3 patients with mutant $P P A R G$, adipose tissue repartitioning had been proposed to explain, at least partially, insulin resistance and hypertension, largely through analogy with other lipodystrophies of different molecular etiologies, since insulin resistance is a prominent component of each of these (6). In lipodystrophies, reduced fat storage capacity has been thought to result in increased circulating fatty acids and ectopic triglyceride storage in such sites as skeletal muscle, leading to insulin resistance with consequent development of complications, including hypertension. However, Tsai et al. show that $\mathrm{Pparg}^{\mathrm{P465L/+}}$ mice did not develop significant insulin resistance (7), in contrast to the severe insulin resistance seen in human PPARG $G^{P 467 L}$ heterozygotes (8). This disparity might be due to a species difference in which human PPAR $\gamma$ retains a more direct link with insulin resistance. Also, the greater relative loss of adipose tissue in human $P P A R G^{P 467 L}$ heterozygotes compared with $P$ parg ${ }^{P 465 L /+}$ mice suggests that adipose tissue loss might still contribute to insulin resistance in human PPAR $\gamma$ deficiency.

On closer inspection, however, the extent of lipodystrophy among patients with mutant PPARG similarly seems insufficient to account fully for the severity of insulin resistance. In this regard, it is instructive to compare FPLD3 with another autosomal dominant form of partial lipodystrophy, namely FPLD2 (OMIM 151660), which results from mutations in $L M N A$ encoding nuclear lamin A/C (6). Like patients with FPLD3, FPLD2 patients have site-specific adipose tissue loss, followed by insulin resistance with hypertension and dyslipidemia, which become even worse as patients age and develop T2DM (12). However, analysis of metabolic subphenotypes indicated that fat loss was more extensive among patients with mutant LMNA (FPLD2) than those with mutant PPARG (FPLD3) (13). In contrast, insulin resistance and hypertension were more severe among patients with FPLD3 than those with FPLD2 (13). Thus, insulin resistance and hypertension in FPLD3 seemed to be disproportionate to the extent of lipodystrophy compared with FPLD2, which would be consistent with additional independent effects of mutant PPARG $(6,13)$. The findings in the $P \operatorname{parg}^{P 465 L /+}$ mice further weaken the case for direct links among adipose redistribution, insulin resistance, and hypertension (Table 1). Could the mutation itself directly mediate hypertension?

\section{PPAR $\gamma$ modulates the renin- angiotensin system}

Tsai et al. (7) show that the PPARG mutation independently affects other pathways, in particular the renin-angiotensin system (RAS). Both human PPARG $G^{P 467 L}$ heterozygotes and Pparg ${ }^{P 465 /{ }^{+}}$mice are hypertensive, despite the fact that the mice are minimally insulin resistant. The hypertension in the Pparg ${ }^{P 465 L /+}$ mice is associated with increased expression of RAS components in various adipose depots, specifically angiotensinogen and the angiotensin II (ATII) receptor subtype 1 in inguinal and gonadal fat, respectively (7). This suggests that impaired adipogenesis might locally activate the RAS, with a potential paracrine role for ATII. Alternatively, mutant PPAR $\gamma$ might have other effects on vascular tone. In any event, the findings suggest a more direct role for PPAR $y$ in blood pressure regulation, possibly through linkage with the RAS. Such a link could be one reason why blood pressure decreases with thiazolidinedione treatment (14) and also why hypertensive heterozygotes for the PPARG loss-of-function mutation F388L respond well to angiotensin-converting enzyme inhibitors (10).

Thus, Tsai et al. have provided novel insights that advance our understanding of PPAR $\gamma$ physiology (7). In both humans and mice, heterozygous PPAR $\gamma$ mutations are associated with lipodystrophy, but in the mouse there is apparently an uncoupling between the adipose repartitioning and hypertension. Furthermore, the hypertension in the Pparg ${ }^{\mathrm{P} 465 \mathrm{~L} /+}$ mice might be functionally linked with RAS 
activity in adipose tissue. The studies cannot resolve whether human PPARG mutations in FPLD3 might act in a dominant negative manner to interfere with function of the normal allele product or whether haploinsufficiency of PPAR $\gamma$ activity is more important. Interestingly, simple haploinsufficiency of PPAR $\gamma$ activity in mice by removal of one Pparg allele actually protects against insulin resistance (15), supporting the idea that missense mutations have distinct effects compared with simple reduction in PPAR $\gamma$. In any event, the findings of Tsai et al. reinforce the importance of PPAR $\gamma$ in adipogenesis (4), highlight the role of adipose tissue as an endocrine organ (16), and also support the idea that PPARG mutations affect metabolic and vascular phenotypes through multiple mechanisms, some of which are distinct from effects on adipose tissue mass or distribution.

Address correspondence to: Robert A. Hegele, Blackburn Cardiovascular Genetics Laboratory, Robarts Research Institute, 406-100 Perth Drive, London,
Ontario N6A 5K8, Canada. Phone: (519) 663-3461: Fax: (519) 663-3037; E-mail: hegele@robarts.ca.

1. Laaksonen, D.E., et al. 2002. Metabolic syndrome and development of diabetes mellitus: application and validation of recently suggested definitions of the metabolic syndrome in a prospective cohort study. Am. J. Epidemiol. 156:1070-1077.

2. Lakka, H.M., et al. 2002. The metabolic syndrome and total and cardiovascular disease mortality in middle-aged men. JAMA. 288:2709-2716.

3. Expert Panel on Detection, Evaluation, and Treatment of High Blood Cholesterol in Adults. 2001. Executive summary of the third report of the National Cholesterol Education Program (NCEP) expert panel on detection, evaluation, and treatment of high blood cholesterol in adults (Adult Treatment Panel III). JAMA. 285:2486-2497.

4. Marx, N., Duez, H., Fruchart, J.C., and Staels, B. 2004. Peroxisome proliferator-activated receptors and atherogenesis: regulators of gene expression in vascular cells. Circ. Res. 94:1168-1178.

5. Stumvoll, M., and Haring, H.U. 2002. Glitazones: clinical effects and molecular mechanisms. Ann. Med. 34:217-224.

6. Hegele, R.A. 2003. Monogenic forms of insulin resistance: apertures that expose the common metabolic syndrome. Trends Endocrinol. Metab. 14:371-377.

7. Tsai, Y.-S., et al. 2004. Hypertension and abnormal fat distribution but not insulin resistance in mice with P465L PPAR $\gamma . J$. Clin. Invest. 114:240-249. doi:10.1172/JCI200420964.

8. Barroso, I., et al. 1999. Dominant negative muta- tions in human PPARgamma associated with severe insulin resistance, diabetes mellitus and hypertension. Nature. 402:880-883.

9. Agarwal, A.K., and Garg, A. 2002. A novel heterozygous mutation in peroxisome proliferatoractivated receptor-gamma gene in a patient with familial partial lipodystrophy. J. Clin. Endocrinol. Metab. 87:408-411.

10. Hegele, R.A., et al. 2002. PPARG F388L, a transactivation-deficient mutant, in familial partial lipodystrophy. Diabetes. 51:3586-3590.

11. Savage, D.B., et al. 2003. Human metabolic syndrome resulting from dominant-negative mutations in the nuclear receptor peroxisome proliferator-activated receptor-gamma. Diabetes. 52:910-917.

12. Hegele, R.A., et al. 2003. Elevated serum C-reactive protein and free fatty acids among nondiabetic carriers of missense mutations in the gene encoding lamin $\mathrm{A} / \mathrm{C}(L M N A)$ with partial lipodystrophy. Arterioscler. Thromb. Vasc. Biol. 23:111-116.

13. Hegele, R.A. 2004. Phenomics, lipodystrophy and the metabolic syndrome. Trends Cardiovasc. Med. 14:133-137.

14. Dobrian, A.D., Schriver, S.D., Khraibi, A.A., and Prewitt, R.L. 2004. Pioglitazone prevents hypertension and reduces oxidative stress in diet-induced obesity. Hypertension. 43:48-56.

15. Miles, P.D., Barak, Y., He, W., Evans, R.M., and Olefsky, J.M. 2000. Improved insulin-sensitivity in mice heterozygous for PPAR-gamma deficiency. J. Clin. Invest. 105:287-292.

16. Engeli, S., et al. 2003. The adipose-tissue reninangiotensin-aldosterone system: role in the metabolic syndrome? Int. J. Biochem. Cell Biol. 35:807-825.

\title{
Innate immunity dictates cytokine polarization relevant to the development of pulmonary fibrosis
}

\author{
Robert M. Strieter and Michael P. Keane
}

\author{
Departments of Medicine, Pathology, and Pediatrics, Division of Pulmonary and Critical Care Medicine, \\ The David Geffen School of Medicine at the University of California, Los Angeles, Los Angeles, California, USA.
}

\begin{abstract}
New data support the importance of the innate immune response in the resolution or progression of pulmonary fibrosis. The presence of CXC chemokine receptor 3-expressing cells, specifically pulmonary NK cells, is necessary to produce IFN- $\gamma$. This is critical in the polarization of the immune response to injury toward a favorable Th1 response and resolution. In contrast, a Th2 response is associated with progressive fibrosis (see the related article beginning on page 291).
\end{abstract}

Pulmonary fibrosis is a host response to a variety of known and idiopathic processes and is clinically characterized by insidious onset of dyspnea and abnormal lung func-

Nonstandard abbreviations used: bronchoalveolar lavage (BAL); CXC chemokine receptor 3 (CXCR3); idiopathic pulmonary fibrosis (IPF); usual interstitial pneumonia (UIP).

Conflict of interest: The authors have declared that no conflict of interest exists.

Citation for this article:

J. Clin. Invest. 114:165-168 (2004).

doi:10.1172/JCI200422398 tion. The pathology of pulmonary fibrosis demonstrates features of dysregulated and abnormal repair with exaggerated vascular remodeling, fibroproliferation, and deposition of extracellular matrix, all leading to progressive fibrosis and loss of lung function. However, the most devastating form is idiopathic pulmonary fibrosis (IPF). IPF is a chronic and often fatal pulmonary disorder with a mortality rate as high as $70 \%$ five years after diagnosis $(1,2)$. The prevalence of IPF increases with age (2, $3)$. There has been an increase in the inci- dence of this disorder over the last 10-15 years in many Western countries, including the United Kingdom, New Zealand, and Germany (3). The inability of current immunosuppressive therapies to alter the prognosis of this disorder has renewed scientific interest in multiple areas and has raised additional questions as follows: (a) why are certain individuals susceptible to this disease? do genetic, environmental, or a combination of these factors contribute to the pathogenesis of this disorder? (b) why is the host response to injury to the alveolar capillary wall in susceptible individuals predominated by fibrosis and loss of alveolar architecture instead of resolution? and (c) what are the initial events within the host in response to injury that ultimately set the stage for polarization of the response toward either resolution or perpetuation of fibrosis? 